\title{
Generation and Visualization of Four-Dimensional MR Angiography Data Using an Undersampled 3-D Projection Trajectory
}

\author{
Jing Liu*, Michael J. Redmond, Ethan K. Brodsky, Andrew L. Alexander, Aiming Lu, Francis J. Thornton, \\ Michael J. Schulte, Thomas M. Grist, James G. Pipe, and Walter F. Block
}

\begin{abstract}
Time-resolved contrast-enhanced magnetic resonance (MR) angiography (CE-MRA) has gained in popularity relative to $\mathrm{X}$-ray Digital Subtraction Angiography because it provides three-dimensional (3-D) spatial resolution and it is less invasive. We have previously presented methods that improve temporal resolution in CE-MRA while providing high spatial resolution by employing an undersampled 3-D projection (3D PR) trajectory. The increased coverage and isotropic resolution of the 3D PR acquisition simplify visualization of the vasculature from any perspective. We present a new algorithm to develop a set of time-resolved 3-D image volumes by preferentially weighting the 3D PR data according to its acquisition time. An iterative algorithm computes a series of density compensation functions for a regridding reconstruction, one for each time frame, that exploit the variable sampling density in 3D PR. The iterative weighting procedure simplifies the calculation of appropriate density compensation for arbitrary sampling patterns, which improve sampling efficiency and, thus, signal-to-noise ratio and contrast-to-noise ratio, since it is does not require a closed-form calculation based on geometry. Current medical workstations can display these large four-dimensional studies, however, interactive cine animation of the data is only possible at significantly degraded resolution. Therefore, we also present a method for interactive visualization using powerful graphics cards and distributed processing. Results from volunteer and patient studies demonstrate the advantages of dynamic imaging with high spatial resolution.
\end{abstract}

Index Terms-Animation, image reconstruction, magnetic resonance imaging, temporal filtering.

Manuscript received July 28, 2005; revised November 1, 2005. This work was supported in part by the National Institutes of Health (NIH) inder Grant 8 R01 EB002075-06, and in part by the Whitaker Foundation, General Electric Medical Systems, and Hewlett-Packard Company. The Associate Editor responsible for coordinating the review of this paper and recommending its publication was E. Bullitt. Asterisk indicates corresponding author.

*J. Liu is with the Department of Electrical and Computer Engineering, University of Wisconsin-Madison. E3/311 Clinical Sciences Center, 600 Highland Avenue, Madison, WI 53792-3252 USA (e-mail: jingliu@ wisc.edu).

M. J. Redmond and A. Lu are with the Department of Biomedical Engineering, University of Wisconsin-Madison, Madison, WI 53706 USA.

E. K. Brodsky and M. J. Schulte are with the Department of Electrical and Computer Engineering, University of Wisconsin-Madison, Madison, WI 537923252 USA

A. L. Alexander is with the Department of Medical Physics, University of Wisconsin-Madison, Madison, WI 53792 USA.

F. J. Thornton is with the Department of Radiology, University of WisconsinMadison, Madison, WI 53226 USA.

T. M. Grist is with the Department of Radiology and the Department of Medical Physics, University of Wisconsin-Madison, Madison, WI 53792 USA.

J. G. Pipe is with the Barrow Neurological Institute, Phoenix, AZ 85013 USA

W. F. Block is with the Department of Biomedical Engineering and the Department of Medical Physics, University of Wisconsin-Madison, Madison, WI 53792-3252 USA (e-mail: wfblock@wisc.edu).

Digital Object Identifier 10.1109/TMI.2005.861706

\section{INTRODUCTION}

C ONVENTIONAL contrast-enhanced magnetic resonance angiography (CE-MRA) studies of the chest and abdomen produce a single image volume timed to capture the first pass of a contrast agent for maximum arterial enhancement [1]-[4]. However, complex flow patterns and retrograde flow can result as an adaptation to vessel occlusive disease in the abdomen or chest. Thus, while a single image volume may be adequate to image some patients, time-resolved MRA capabilities are necessary to visualize the vasculature in many cases. Time-resolved methods are also of increasing interest because they can simplify exams by mitigating the need to precisely coordinate imaging and contrast arrival [5], [6].

Time-resolved 3-D MR data [x, y, z, and time or four-dimensional (4-D)] can be generated by repetitively acquiring spatial frequency (k-space) data [7], [8]. However, spatial resolution or coverage must be significantly sacrificed for modest frames rates due to the relative slow speed of MR acquisition and the necessity of a breath-hold scan. Oversampling the center of k-space can exploit the image contrast generated by the central k-space frequencies, as in time-resolved imaging of contrast kinetics (TRICKS) [9]. To date, the clinical applications have been shown primarily in the peripheral vasculature and to a lesser extent in the carotid arteries. The limited scan time afforded by the breath-hold requirement in the abdomen and chest and the desire for high resolution has limited techniques that redundantly sample Cartesian k-space.

We have previously shown that the high image contrast between vessels and background in CE-MRA allows accelerated imaging using an undersampled 3-D projection (3D PR) trajectory [10], known as vastly undersampled isotropic projection (VIPR) imaging [11]-[13]. The trajectory generates a spherical field of view (FOV) covering the entire torso, similar to computed tomography (CT) and, thus, increases coverage while simplifying scan prescription. Since the sampling strategy is equivalent in each spatial dimension, resolution is isotropic and the imaging volume can be viewed from any perspective equally well. The artifacts from undersampling 3D PRs resemble structured noise which affects the achievable acceleration factor. This factor increases with the available image contrast and as the number of objects creating strong signal decreases. Both of these factors are favorable in CE-MRA where the vessel signal is high and most nonvascular tissue has low signal.

Since the center of k-space is repeatedly sampled during each excitation, a conventional reconstruction which equally weights 
data from each excitation will output a time-averaged representation of contrast enhancement during the scan. We have previously demonstrated the generation of time-resolved imaging volumes from VIPR exams, including a peak arterial time frame [10]. By filtering the data based on the time of acquisition, the variable sampling density of 3D PR may be exploited. The width of the temporal filter varies with spatial frequency, narrowing to selectively emphasize lower, oversampled spatial frequencies from a desired time interval. The shape of the filter was empirically designed. However, time-resolved imaging showed image quality degradation including flicker artifacts not present in the standard reconstruction. Also, the minimum scan time was $40 \mathrm{~s}$ and, thus, was too long for many patient exams.

We present here a general, deterministic algorithm to generate temporal filters for variable density k-space acquisitions and apply these to the VIPR acquisition. The temporal filtering is incorporated into an algorithm to compensate for variable sampling density trajectories. While our previous design required inputs to specify the filter shape for all spatial frequencies, this method only requires one temporal input curve. In addition, the design properly calculates the density compensation function for more complicated trajectories, which may enable the scan time to be shortened.

When reviewing these exams, the added time dimension and anatomical coverage increase the required memory for visualization tools by a factor of 15 to 20 . Current medical workstations provide some capability for large exams, but only with a substantial loss of resolution at very modest frame rates during interactive operations. We present a method for interactively visualizing 4-D data with no loss in resolution using powerful graphics cards and distributed processing.

The 4-D imaging is demonstrated through clinical exams on patients whose conditions make single time frame visualization inadequate. These conditions include slow-filling aneurysms, aortic dissections, and complex flow networks.

\section{THEORY}

\section{A. Three-Dimensional PR Acquisition and General Reconstruction}

Three-dimensional PR data are acquired on a spherical coordinate system [14], with the readout direction defined by the azimuthal and elevation angle, as shown in Fig. 1(a). The maximum $\mathrm{k}$-space radius value $\left(\mathrm{k}_{\max }\right)$ determines the spatial resolution. The radial sample spacing $\left(\Delta \mathrm{k}_{\mathrm{r}}\right)$ determines the diameter of the full FOV. To shorten scan time, this implementation of VIPR collects multiple radial lines per repetition time (TR), as shown in Fig. 1(b) [15]. Each radial line corresponds to a half-echo. The corresponding gradient waveforms to cover this trajectory are shown in Fig. 1(c) while the endpoints of all the sampled radial lines are derived from a waveform that samples the surface of a sphere, as shown in Fig. 1(d). A gradient calibration scheme is used so that signal from each radial line within a TR will add coherently [16]. Further details of the acquisition are beyond the scope of this paper.

The point spread function contains a properly reconstructed impulse in the center that is surrounded by a region free from artifact [17], [18]. Artifact energy is located outside of this region.

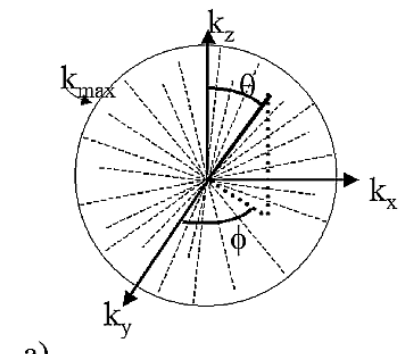

a)

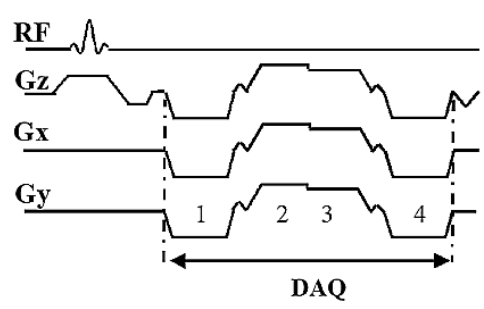

c)

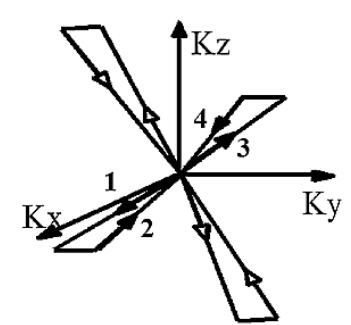

b)

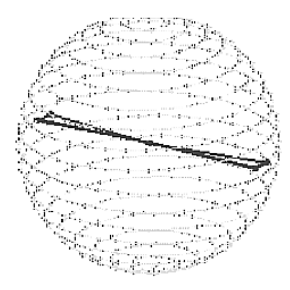

d)
Fig. 1. (a) Radial lines are acquired in 3-D k-space at an angle $\phi$ in the transverse plane and angle $\theta$ from the longitudinal axis. (b) During each TR, four radial lines are acquired to increase data acquisition efficiency. The numbered radial lines (1-4) acquired in one repetition time (TR) are shown with black arrows. Data acquired during another TR are shown with white arrows. The trajectory forms the shape of a bowtie each TR. (c) Pulse sequence diagram uses a gradient-recalled echo sequence with four marked lobes, corresponding to the lines in (b). (d) Endpoints of the radial lines sample the surface of a sphere. Here the samples within one TR are shown.

The radius of this alias-free region, as predicted by the Nyquist sampling criteria, is $(1 / 2) \sqrt{N_{p} / \pi}$, where $N_{p}$ is the number of radial lines sampled. A fully sampled object requires $\pi N_{r}^{2}$ radial lines, where $N_{r}$ is the number of k-space samples along each radial line.

Image reconstruction is achieved by regridding or interpolating the spherical coordinate k-space data onto a Cartesian grid [19], performing an inverse Fast Fourier Transform (FFT), and then deappodizing the image to remove the intensity modulation caused by interpolation in k-space. A density compensation function is required during the regridding operation if the sampling density is not uniform. The design of the density compensation function is a major focus of this work. Although the function is discussed here as a portion of the reconstruction process, it is independent of the acquired data values and can be computed beforehand and used for all scans with the same k-space trajectory.

\section{B. Density Compensation for Time-Averaged Imaging}

The sampling density theoretically varies as $1 / k_{\rho}^{2}$ for $3 \mathrm{D} \mathrm{PR}$, where $k_{\rho}$ is the k-space radius. The theoretical weighting compensation function is, thus, $k_{\rho}^{2}$. Creating a sampled space whose density does not vary as a function of the azimuth and elevation coordinates is difficult, however. Also, the multiple radial line sequence, shown in Fig. 1(c), acquires faster by sampling during periods of variable gradient amplitude and, thus, k-space velocity. Therefore, the compensation function is more complex than $k_{\rho}^{2}$.

To develop a density compensation function for more complicated trajectories, we modified an iterative algorithm proposed by Pipe et al. [20] for undersampled 3-D trajectories. We first 
present the algorithm for time-averaged imaging, which will serve as a foundation and necessary intermediate calculation for the time-resolved imaging.

An iterative solution for the density compensation function, $W$, may be used whose solution

$$
W_{i+1}=\frac{W_{i}}{\left(W_{i} \otimes C\right) \bullet S}
$$

minimizes the least square error in the point spread function [20]. Here $S$ represents the sampled points within the k-space trajectory, $C$ is the convolution kernel used during regridding, and $W$ is only nonzero at the sampled points. The periodicity of 3-D radial imaging allows a simple calculation of an initial weight function instead of using the function suggested in [20]. The initial weight function was calculated as the square of the $\mathrm{k}$-space radius times the magnitude of the gradient vector, a normalizing factor for varying k-space velocity during any possible ramp sampling.

A circularly symmetric Kaiser Bessel function whose radius was equal to $1 / \mathrm{FOV}$ can be used as the convolution kernel [21]. When undersampling causes sampling intervals to be greater than $1 / \mathrm{FOV}$, this algorithm views the sampled points as uncorrelated and calculates uniform density compensation as compared to the theoretical $k_{\rho}^{2}$. This weighting decreases the mean-squared error but also reduces spatial resolution. Higher resolution may be obtained by using a wider convolution kernel when calculating the density compensation function [22]. The ratio by which the convolution function radius must be enlarged for maximum resolution is $\sqrt{\pi N_{r}^{2} / N_{p}}$.

This density compensation for each $\mathrm{k}$-space point is independent of its time of acquisition and, thus, this function produces a time-averaged representation of enhancement during the scan. We will refer to this density compensation, an important intermediate calculation for time-resolved imaging, as $W_{t a}$ where the subscript $t a$ stands for time-average.

\section{Density Compensation for Time-Resolved Imaging}

As shown earlier in Fig. 1(d), the endpoints of the radial lines sample the surface of the sphere. The acquisition is not ordered to move monotonically from the bottom pole of the sphere to the top pole or to sweep along lines of constant longitude. Rather, the order of acquisition is interleaved to sample directions throughout the entire k-space sphere $N$ times at intervals, $\Delta t$, such that the entire scan time is $N \Delta t$. Thus, at least some low spatial frequencies are oversampled every $\Delta t \mathrm{~s}$, independent of orientation, whereas higher spatial frequencies are sampled at less frequent intervals.

Let us assume a highly undersampled series of $N$ time-resolved image volumes is to be reconstructed at times $n \Delta t$, where $n$ is an integer varying from 1 to $N$, with a sliding window reconstruction that grids data only from the time interval $(n-1) \Delta t$ to $n \Delta t$. However, for short time intervals, data from one interval alone may have insufficient signal-to-noise ratio (SNR) and contrast-to-noise ratio (CNR) compared to the quality achievable in a single time frame scan of duration $N \Delta t$.

Instead, we aim to develop a density compensation function that preferentially weights oversampled data acquired closer to the desired time point, $n \Delta t$, of each time-resolved image volume.
Since the k-space density decreases rapidly in 3D PR as a function of the radial coordinate $k_{\rho}$, the temporal filter should ideally widen as $k_{\rho}$ increases. At radii where $\mathrm{k}$-space is undersampled, the compensation function should use undersampled data across all time intervals to minimize the undersampling artifact.

An iterative density compensation equation was previously developed to weight a nonuniform trajectory where patient motion may alter the relative worth of redundantly sampled data [23]. Here we modify this method to create a weighting biased toward the time interval from $(n-1) \Delta t$ to $n \Delta t$, referred to as $W_{t r}(n)$, by first solving two iterations of (2)

$$
W_{k+1}(n)=\frac{W_{k}(n)}{\left(\left(W_{k}(n) \bullet F_{n}(t)\right) \otimes C\right) \bullet S} .
$$

Here the density compensation function for data acquired along one radial line is weighted by a single value, $F_{n}(t)$, where $t$ is the point during the scan at which the line is acquired. $F_{n}(t)$, a one-dimensional (1-D) function, weights data more heavily if they are acquired closer to the $n$th time frame. The initial approximation in (2), $W_{k=0}$, is the time-averaged solution provided by (1), $W_{t a}$.

Then the solution $W_{k+1}(n)$ is substituted into (3) to produce $W_{t r}(n)$

$$
W_{t r}(n)=F_{n}(t) \cdot W_{k+1}(n) .
$$

In regions that are oversampled (3) provides the temporal weighting based on the time of acquisition, but (2) is necessary to maintain the condition that $W_{t r}(n) \otimes C=1$. In regions where the trajectory is undersampled, the weighting $W_{t r}(n)$ tends toward $W_{t a}$ since the initial approximation will decrease by $1 / F_{n}(t)$ in (2) and then be increased by $F_{n}(t)$ in (3). Notice also that a constant $F_{n}(t)$ reduces the solution to (1), the time-averaged solution $W_{t a}$.

Thus, the problem of designing the density compensation $W_{t r}(n)$ does not require specifying the temporal aperture as a function of spatial frequency. Instead, the only input is a user-defined function $F_{n}(t)$ that describes how points at the k-space origin, sampled in each radial line, should be weighted. The algorithm automatically calculates the density compensation for other spatial frequencies. The algorithm aims to use the function $F_{n}(t)$ to weight the projections at spatial frequencies where there is sufficient oversampling. As the degree of oversampling decreases with increasing $k_{\rho}$, the algorithm effectively widens $W_{n}(t)$. The algorithm then scales this 1-D weighting function to other spatial frequencies based on their time of acquisition and degree of oversampling.

The shape of $F_{n}(t)$ generally determines the tradeoff between SNR and temporal bandwidth, with better temporal resolution resulting for the case where $F_{n}(t)$ is a sharper, narrower function. However, the SNR for static objects is optimal when $F_{n}(t)=1$. For a specific object with a time-variable intensity $i(t)$, designing a filter with optimal SNR for the object is beyond the scope of this paper. While a matched filter implementation where $F(t)=i(t)$ will optimize signal from one object, it may create more deleterious artifacts from other objects.

As an analog to time-resolved imaging, methods have been proposed to create multiple $\mathrm{T} 2$-weighted images from one two- 
dimensional (2-D) radial dataset acquired over a long echo train [24], [25]. In these schemes, k-space was segmented into three or four distinct annuli. As the radius of the annuli decreased, fewer radial lines were needed to represent each region properly. For each specific T2-weighting, only the radial lines acquired during the corresponding portion of the echo train were used. These techniques have some similarities to the reduced FOV radial imaging techniques [26] and our previous implementation [10]. To our knowledge the algorithm described in this paper is the first that allows a continuous weighting of the oversampled data with proper density compensation. The design of $F_{n}(t)$ is described later in the Section III.

\section{METHODS}

MRA scans are acquired by a technologist as part of a larger exam. Multiple patient exams are typically reviewed later by a radiologist using a computer workstation. Angiography exams are visualized using a Multiplanar Volume Reformat (MPVR) tool that allows one to interactively restrict analysis to select a subset of the imaging volume. Vessels within this region are interactively visualized through a maximum intensity projection (MIP) from various viewing perspectives. After a subvolume is examined, a new subvolume can be selected to study other vessels. Since the diagnostic review is done independently of the scan and since the time of the radiologist impacts revenue, the visualization process must be rapid while the time for the reconstruction process is more flexible.

\section{A. VIPR Acquisition Implementation}

The imaging sequence was implemented as a T1-weighted, $\mathrm{RF}$ spoiled gradient recalled sequence on a 1.5-T General Electric CVi scanner (GE Healthcare, Milwaukee, Wisconsin) equipped with high-performance gradients $(40 \mathrm{mT} / \mathrm{m}$ peak amplitude and $150 \mathrm{mT} / \mathrm{m} / \mathrm{ms}$ peak slew rate). During each repetition time (TR) of $4.4 \mathrm{~ms}$, four radial lines were acquired over $2.4 \mathrm{~ms}$ using a $+/-125 \mathrm{kHz}$ receiver bandwidth, a maximum radial extent in $\mathrm{k}$-space of 0.29 cycles $/ \mathrm{mm}$ ( $1.7 \mathrm{~mm}$ resolution), a FOV of $44 \mathrm{~cm}$, and a $30^{\circ}$ flip angle. The trajectory within each TR, as shown in Fig. 1(b), sampled the origin of k-space at echo times of $0.3 \mathrm{~ms}, 1.3 \mathrm{~ms}$, and $2.4 \mathrm{~ms}$. The radial lines were interleaved to sample orientations throughout the k-space sphere at an interval, $\Delta t=2 \mathrm{~s}$.

Institutional board approval and informed consent were obtained for 7 volunteers and 13 patients. An antero-posterior phased array torso surface coil with either 4 or 8 elements was placed over either the chest or the abdomen. An intravenous injection of 25-40 cc gadopentetate dimeglumine (Omniscan, GE Healthcare, Milwaukee, WI) was administered with an automated power injector (Spectris; Medrad, Pittsburgh, PA) at 4.0 to $5.0 \mathrm{ml} / \mathrm{s}$. Data acquisition began approximately $4 \mathrm{~s}$ after the start of the injection in pulmonary studies and $10 \mathrm{~s}$ after injection in abdominal studies. Patients were requested to hold their breath for $30 \mathrm{~s}$, but encouraged to simply exhale slowly if the breath-hold duration was too demanding. Each scan acquires projections on approximately 30000 radial lines. None of the exams used fluoroscopic triggering or a timing scan to predict the arrival of contrast. Rather rough rules of thumb were used to start the scan shortly before the arrival of contrast.
A regridding reconstruction was used to interpolate the k-space data onto a $256^{3}$ Cartesian matrix prior to an inverse Fourier transform. A separate reconstruction with a different density compensation function $W_{t r}(n)$ [(2) and (3)] was used to reconstruct the image volume at each time interval. The processing time was approximately $20 \mathrm{~s} /$ receiver coil per time interval using a dual processor $1.4 \mathrm{GHz}$ Athlon PC with 1 Gbyte of RAM. We next discuss in further detail the calculation of the density compensation function $W_{t r}(n)$.

\section{B. Density Compensation Computation}

The convolution in the denominator of $(1),\left(W_{i} \otimes C\right) \bullet S$, is costly to calculate. Since the sampled points are radially oriented and distribute unevenly during the ramp sampling, selecting the points in $S$ which must be queried to determine if they are within the limited width of the convolution kernel $C$ is more difficult. An approximation of $\left(W_{i} \otimes C\right) \bullet S$ can be obtained much faster by interpolating $W$ onto a sufficiently dense Cartesian grid $R$ with the convolution kernel $C^{1 / 2}$, and then interpolating back onto $S$ using the same kernel $C^{1 / 2}$ [20]

$$
\left(W_{i} \otimes C\right) \bullet S=\left(\left(\left(W_{i} \otimes C^{\frac{1}{2}}\right) \bullet R\right) \otimes C^{\frac{1}{2}}\right) \bullet S
$$

where $C^{1 / 2}$ denotes the square root value of the convolution $C$. This procedure reduces computation cost from $\mathrm{O}\left(N^{2}\right)$ to $\mathrm{O}\left(N L^{2}\right)$, where $N$ is the number of samples and $L$ is the width of the convolution function. Since $N$ is on the order of $10^{6}$ and $L$ is on the order of $2-4$, the computation cost is reduced dramatically.

The approximate initialization of $W_{t a}$ described earlier speeds the convergence of the iterations significantly. The time-averaged weighting $W_{t a}$ was calculated using only two iterations.

\section{Temporal Weighting Function $F_{n}(t)$}

We have chosen the function $F_{n}(t)$ as

$$
\frac{1}{c \sqrt{|\Delta \tau|}}
$$

where $c$ is an application dependent constant and time $\Delta \tau$ is the time frame index relative to the desired time frame within the scan. Since the volume of the k-sphere that is fully sampled grows as the square root of the acquisition time in $3 \mathrm{D} P R$, choosing a weighting that falls off at the inverse of this rate is a logical choice. Sample functions for four time frames, describing the relative weighting of each time interval of k-space, are shown in Fig. 2.

As the constant $c$ decreases, SNR improves due to signal averaging at lower spatial frequencies but also causes a tradeoff of decreased temporal resolution. The constant $c$ is chosen to be higher for abdominal scans where renal veins quickly enhance and opacify the viewing of the renal arteries. When temporal resolution is less stringent, as in the peripheral vasculature, a lower $c$ can be used. The constant $c$ was set to 25 to provide adequate venous rejection in the abdominal and thoracic imaging in this paper. For values higher than this, venous suppression improves minimally while considerably more iterations of (2) are necessary for convergence. 


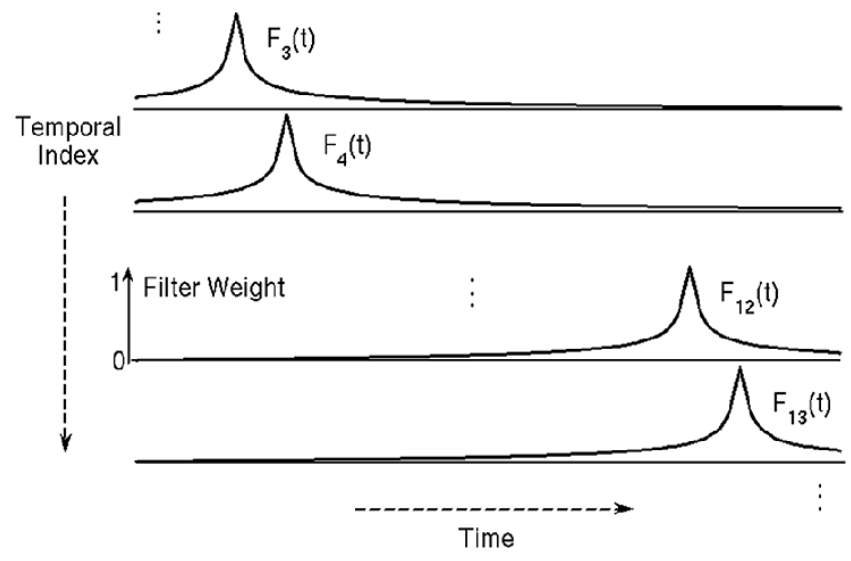

Fig. 2. Temporal weighting functions $F_{n}(t)$ is illustrated for 4 of 15 time frames for a $30-\mathrm{s}$ exam. The sharp falloff provides venous suppression.

Radial lines that are oriented perpendicular to the long axes of vessels have more high spatial frequency energy. To avoid biasing a vessel's intensity based upon its orientation, we set $F_{n}(t)$, according to (5), to be piecewise constant over the time interval of each time frame. In our reconstruction, only two iterations of (2) were used when c was set to 25 .

We illustrated the computed density compensation functions over 3-D k-space as a 2-D gray-scale image, with the k-space radial coordinate $\rho$ of each sample point serving as the vertical axis while the acquisition time of each radial line serves as the horizontal coordinate.

\section{Four-Dimensional Cluster Visualization}

A VIPR abdominal exam can provide 15-25 temporal frames, each with isotropic resolution over a $256^{3}$ image matrix. While cine visualization of these large data sets is possible on medical workstations, simultaneous manipulation and cine presentation usually require reduced manipulation response, playback frame rate and/or image resolution. We present a possible solution in two steps where PC graphics cards first speed processing within a small number of time frames and processing is distributed for larger visualization tasks.

Current workstations exploit the processing power of PC graphics hardware that are separate from the main system CPU and memory. For single frame presentation, we implemented a MPVR tool to utilize these advanced hardware rendering capabilities to directly render limited MIP images from the volumetric data.

Rendering performance is compromised for scenes that are too large to be stored in the dedicated on-board memory of the graphics card. A prototype 4-D Cluster Visualization (4DCV) system [27] was developed to segment the VIPR visualization task across several nodes, as shown in Fig. 3. Since the rendering of each time frame is independent of the others, $4 \mathrm{DCV}$ can distribute the rendering task to provide simultaneous manipulation and rendering. In practice, the display of each node is segmented to render four time frames per node. By interleaving the frames, contention for rendering resources on any one node during sequential animation is minimized.

This approach is competitive when the cost of the initial data load is not a major factor. Thus, a calculated tradeoff for clinical

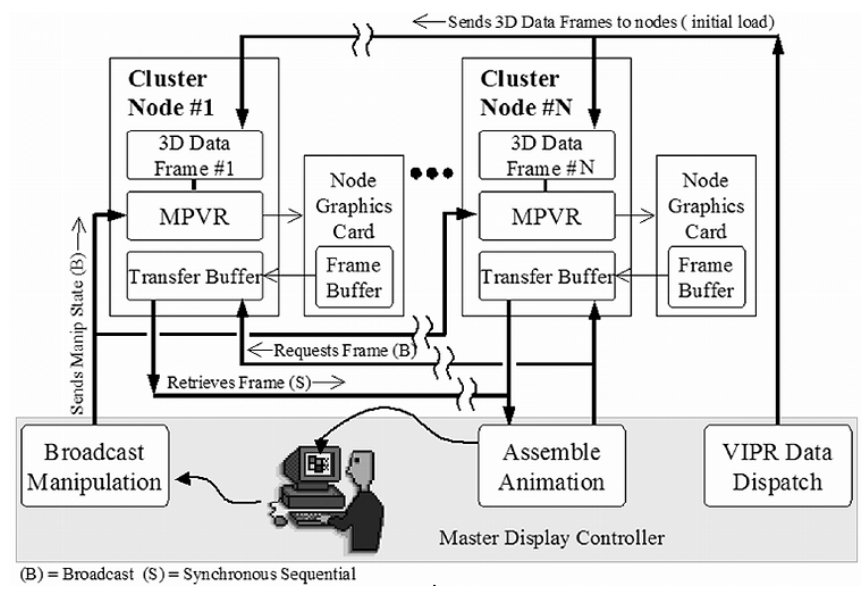

Fig. 3. Four-dimensional cluster visualization functional block diagram illustrated with one time frame per node for simplicity.

evaluations exists, where load times are expected to comprise a small fraction of the total evaluation process.

The 4DCV prototype was developed by creating both master and cluster node functionality for the MPVR tool. The master node tracks user inputs, which specify the viewpoint and volume to be rendered, and then broadcasts these inputs to the cluster nodes. A looping animation is created when the master periodically makes rendering requests to the cluster nodes through the Assemble Animation task labeled in Fig. 3. The cluster nodes read the graphics card frame buffer when requested and send the rendered images back to the animation display process.

The prototype, written in C using OpenGL libraries, was implemented on an Itanium II cluster donated by the Hewlett Packard Company. Each node has $4 \mathrm{G}$ of memory, an NVidia Quadro4 980XGL graphics card, and is interconnected via 100 Mbit switched Ethernet. Subsequent prototypes were successfully implemented on less powerful NVidia Quadro 750XGL graphics cards with $1 \mathrm{G}$ of memory [28].

\section{E. Analysis}

Ten VIPR MRA exams were reviewed by a radiologist with experience in CE-MRA to quantify changes in image quality caused by the temporal filtering. The studies were reconstructed using both the previously described empirical temporal filter design [10] and the iterative filter design described in this paper. Quantitative SNR was measured in the descending aorta, the right renal artery, and in the superior mesenteric artery in the peak arterial frame. SNR was measured as the ratio of the mean signal within a vessel to the mean background region. All studies were also reviewed in a blinded fashion and were graded qualitatively for presence of artifact, vessel visualization, and the perceived temporal resolution of small vessels (mesenteric arteries) relative to large vessels (abdominal aorta). This was performed by interactively visualizing targeted MIPs of $8 \mathrm{~cm}$ in thickness. Presence of artifact was scored visually as the reader visualized time-resolved vessels in a cine loop as follows: $4=$ no artifact, $3=$ minimal artifact, $2=$ mild artifact, $1=$ moderate artifact, and $0=$ severe artifact. Grading of vessel depiction was determined by evaluating the segmental renal arteries, third-order lower lobe pulmonary arteries (peripheral), left 

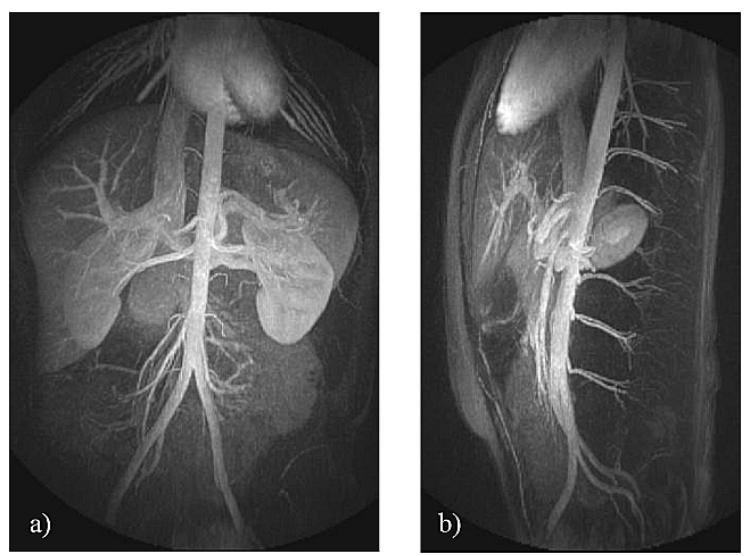

Fig. 4. VIPR abdominal study with time-averaged density compensation shows broad coverage in collapsed views from the (a) coronal and (b) sagittal perspective. Interpretation is difficult, however, due to the overlying arterial and venous structures.

renal vein, portal vein, and mesenteric arteries. Arterial and venous structures were characterized based on a visual score using a 5-point scale where $4=$ excellent visualization, $3=$ slightly limited but good enough to describe pathology, 2 = suboptimal for pathological evaluation, $1=$ minimally perceived, and $0=$ vessel not seen. Temporal resolution was scored based upon the perceived relative change in signal intensity or contrast within the mesenteric arteries (largely high spatial frequency data) relative to the abdominal aorta (largely low spatial frequency data): $4=$ large change, $3=$ moderate change, $2=$ mild change, $1=$ minimal change, and $0=$ absence of change.

\section{RESULTS}

Since fat and water develop a $\pi$ phase difference every $2.2 \mathrm{~ms}$ at $1.5 \mathrm{~T}$, the multiple traversals of the k-space origin provided some beneficial cancellation of off-resonance fat signal. The fat cancellation allows thick reformats showing much more anatomy than in our previous work [10]. Complete coverage of the torso with isotropic resolution is demonstrated in Fig. 4 from the coronal and sagittal perspective. Using the time-averaged density compensation, one can appreciate structures such as the heart, aorta, renal arteries, mesenteric network, renal veins and hepatic network, however, no information is given on the order in which these vessels enhance. The overlying of venous structures over arterial structures makes interpretation of the time-averaged image volume difficult, however. For example, the left renal vein obscures the left renal artery.

Each $W_{t r}(n)$ was calculated using two iterations of (2) and required $40 \mathrm{~s}$ on the Athlon processor described earlier. A typical density compensation function for the sixth of 15 time frames, $W_{t r}(6)$, is illustrated in Fig. 5. Notice that near the $\mathrm{k}$-space origin the weighting function narrows to emphasize data taken primarily during the intended interval, here the sixth time frame. The weighting function widens in time as the $\mathrm{k}$-space radial coordinate increases. Above the dotted line in Fig. 5, k-space is undersampled and there should be no temporal weighting. Although the delineation of the current time frame in the undersampled region is visible, it becomes less apparent with further iterations of the algorithm. However,

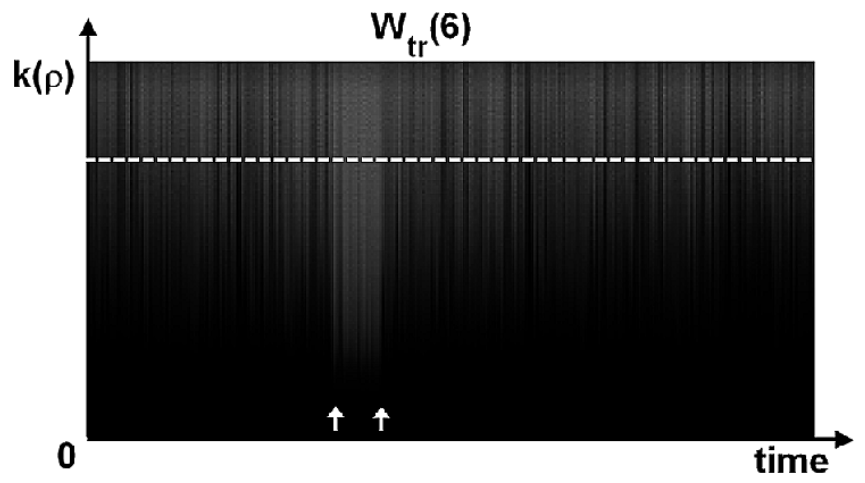

Fig. 5. Time-resolved density compensation function for the sixth of 15 time frames shown as gray scale value. The start and end times of the time frame are shown with white arrows. Three-dimensional k-space is plotted for radial lines acquired with increasing scan time on the horizontal axis while the k-space radial dimension is the vertical coordinate. Above the dotted line, k-space is undersampled and, thus, there is no temporal weighting.

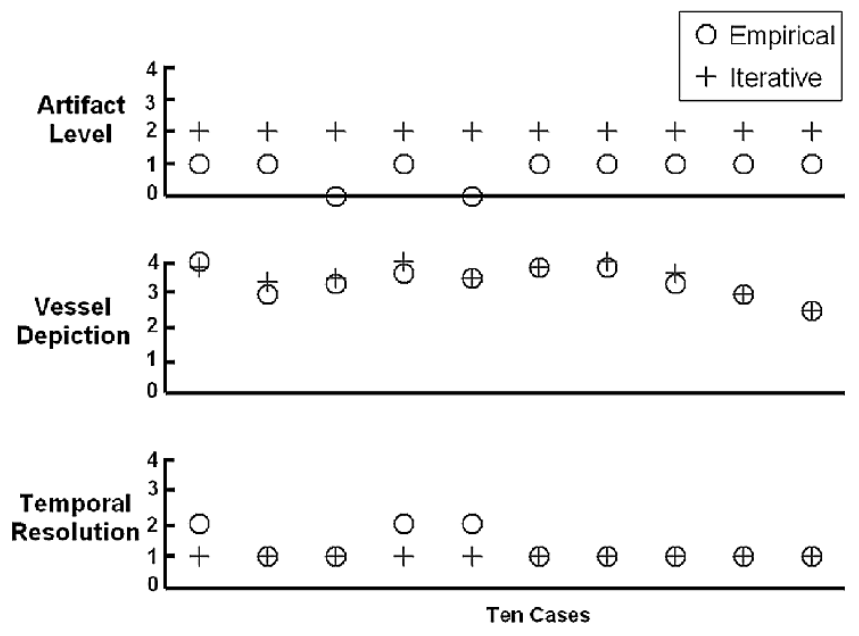

Fig. 6. Grading of artifact level, vessel depiction, and temporal resolution of ten patient cases by using empirical temporal filter design and iterative temporal filter. A significant reduction in artifact level was detected with the iterative filter design. The iterative filter's wider temporal window results a small loss in temporal resolution.

these iterations were not typically performed because they made negligible changes in image quality. The horizontal variation in Fig. 5 over short time intervals demonstrates that achieving equidistant angular intervals is difficult in 3D PR.

One patient exam failed and had to be repeated when contrast took $30 \mathrm{~s}$ to travel from the arm to ascending aorta. Another exam failed when the scanner failed to save the MR data. This problem was solved after updating the scanner's data acquisition subsystem.

Qualitative artifact level, vessel depiction, and the perceived temporal resolution of small vessels (mesenteric arteries) relative to large vessels (abdominal aorta) were graded respect to the descriptions in the Analysis portion of Section III-E. As shown in Fig. 6, a consistent improvement in artifact level was present with 4-D image volumes using the iterative temporal filter design rather than the empirical design. A small improvement in vessel depiction was qualitatively reported in 5 of the 10 cases using the iterative design. No difference was reported in 4 cases and a small decrease was noted in one case. Note that the grade 

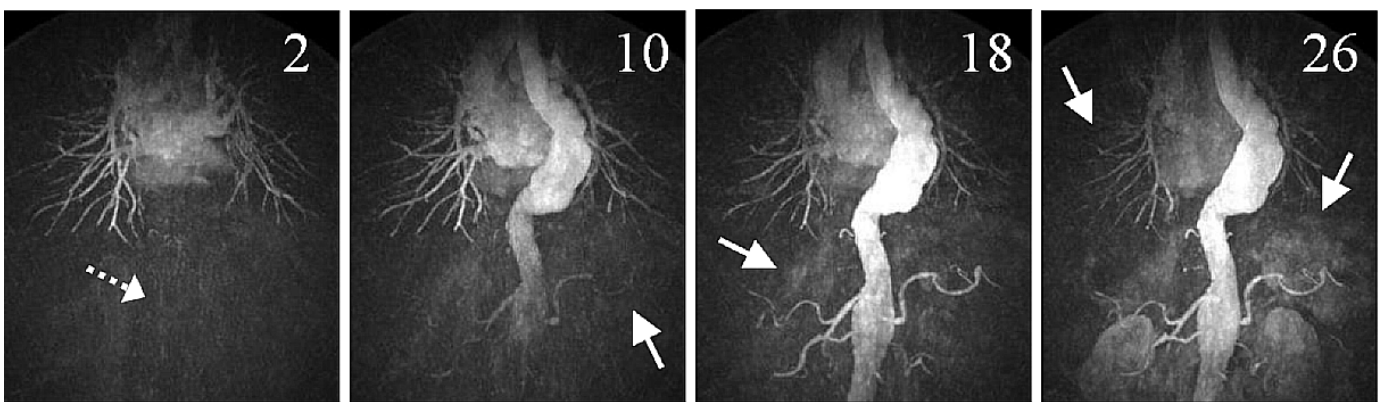

\section{Empirical}
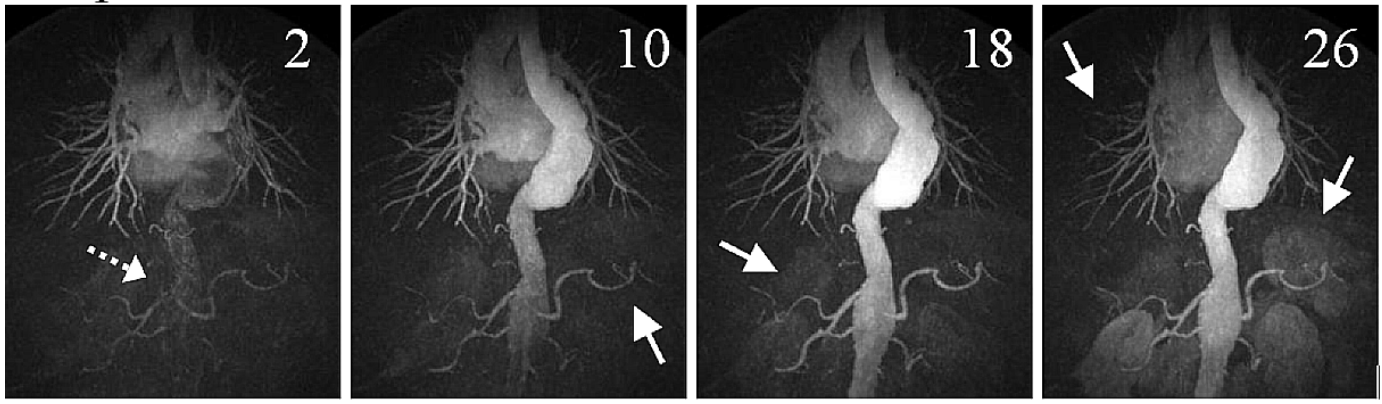

Iterative

Fig. 7. Time-resolved coronal MIPs depict flow at four points at 8-s intervals through an aneurysm (time point are shown in white numbers). Improvement in artifact level is shown by using the proposed iterative temporal filter (bottom row) compared to the previous empirical design (top row). Arrows in second time frames show better depiction of abdominal vessels while those in the third and fourth time frames show less artifact in liver parenchyma and spleen. The early enhanced aorta pointed by the dashed arrows in the first time frames demonstrates a slight loss in temporal resolution.

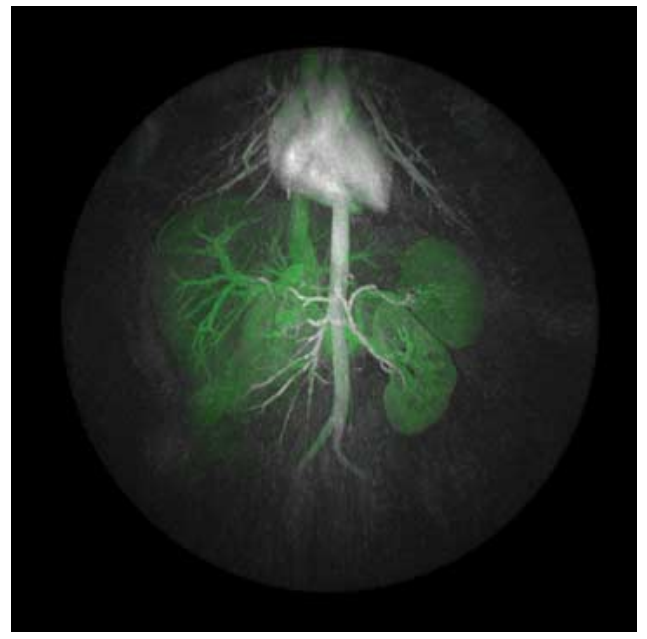

Fig. 8. Abdominal CE-MRA of coronal slab shown from a coronal perspective shows arterial frame (gray) and venous frame (green).

of vessel depiction was averaged over those five evaluated regions of each study case. The expanded use of high spatial frequency data in the iterative filter design caused a small loss in temporal resolution for smaller vessels relative to the empirical design, as also shown in Fig. 6.

The radiologist's criteria for grading vessel depictions were based on providing a clinical diagnosis. As both the empirical and iterative methods graded well in this regard, there was not a significant difference in the qualitatively observed vessel depiction score. However, the iterative temporal filter design demonstrated an average quantitative improvement in SNR of $24.9 \%$, $24.1 \%$, and $13.4 \%$ in the aorta, right renal, and superior mesenteric arteries, respectively.
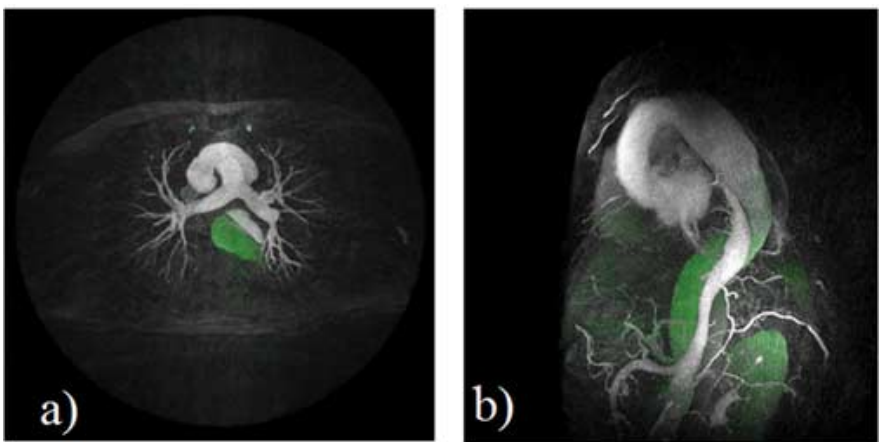

Fig. 9. Aortic dissection with (a) axial view and (b) sagittal view. Gray scale depicts how true lumen enhances first. Regions enhancing later, shown in green, demonstrate false lumen.

In Fig. 7, time frames depicting a descending aortic aneurysm show the lumen of the aorta more clearly using the iterative filter design. ${ }^{1}$ In the second shown time frame, major abdominal vessels (arrows) such as the renal and splenic arteries are better demonstrated. In the third and fourth time frames, considerably less artifact was seen in the liver parenchyma and spleen, as shown by the arrows. The dashed arrows in the first time frame show that the aorta prematurely enhances with the iterative filter, which is further described in the Discussion section. This demonstrates the previously described slight loss in temporal resolution. This case demonstrates that multiple time

${ }^{1}$ This paper has supplementary material available at http://ieeexplore.ieee.org/, provided by the authors. An avi movie shows the filling of the aneurysm in three orthogonal frames for the case shown in Fig. 7. This material is $719 \mathrm{~KB}$ in size. Another mpeg movie shows a rotating MIP about the long axis of the body acquiring during a time of peak arterial enhancement with minimal venous overlay. This material is $1.7 \mathrm{MB}$ in size. 


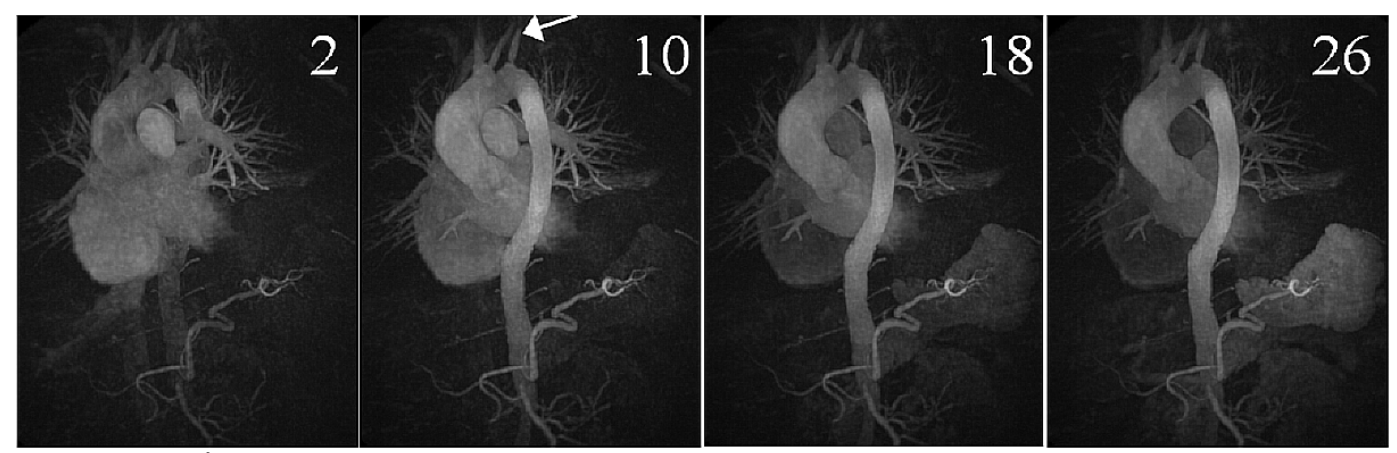

Coronal
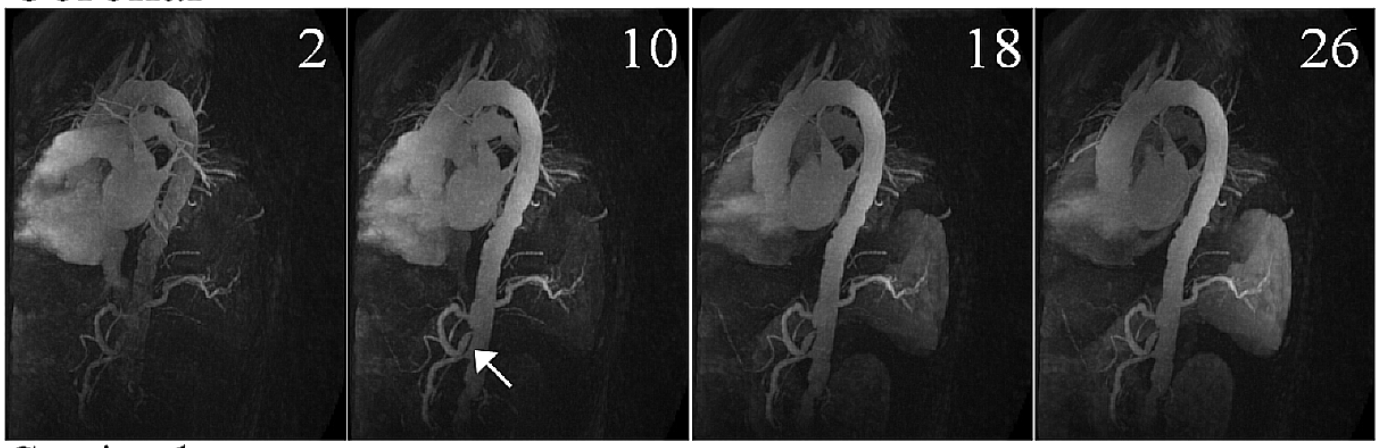

Sagittal

Fig. 10. Time-resolved coronal (top) and sagittal (bottom) MIPs depict flow throughout an exam by spacing four points at 8-s intervals (time point shown in white numbers). Initially, contrast is brightest in the pulmonary arteries and the right side of the heart, but moves later frames to the aorta, and finally the spleen. Stenoses in the subclavian artery (long arrow) and celiac trunk (short arrow) are enlarged in Fig. 11.

frames are necessary to depict the entire vasculature of the abdomen when filling is slow. The multiple time frames allow visualization of the perfusion of the entire kidney as well.

The MPVR tool was used to generate the clinical images shown in this section. Separation of the arterial and venous signal can be achieved by coloring the MIP of the difference of an early and late time frame in green and the MIP of an early frame in grayscale. A reformat from the coronal perspective separating the renal and mesenteric arteries from the hepatic veins is shown in Fig. 8. Green color is used to separate the false lumen from the true lumen, shown in gray scale, for an aortic dissection in Fig. 9.

Progression of contrast from the right side of the heart and pulmonary arteries to the aorta, abdominal vessels, and lastly the spleen are shown in the images of a patient with multiple stenoses in Fig. 10. ${ }^{2}$ Broad anatomical coverage is again shown via both sagittal and coronal reformats. Enlarged regions of the images clearly show stenoses in the subclavian artery and the celiac trunk in Fig. 11. The latest 4DCV prototypes have achieved simultaneous manipulation and animation rates of 10 frames/s. The six-node prototype has been used to display a sequence with 24 frames using four-way display segmentation. The display successfully demonstrated several types of flow patterns with clinical significance. Flow through the heart could be visualized to rule out anomalous pulmonary venous return. Abdominal flow patterns were successfully studied to identify the source of blood in a large vascular network feeding the spleen.

${ }^{2}$ This paper has supplementary material available at http://ieeexplore.ieee.org/, provided by the authors. An mpeg movie clip is available showing $4 \mathrm{DCV}$ performance and operation at the operator's console for the case shown in Fig. 10. This material is 8.7 MB in size.
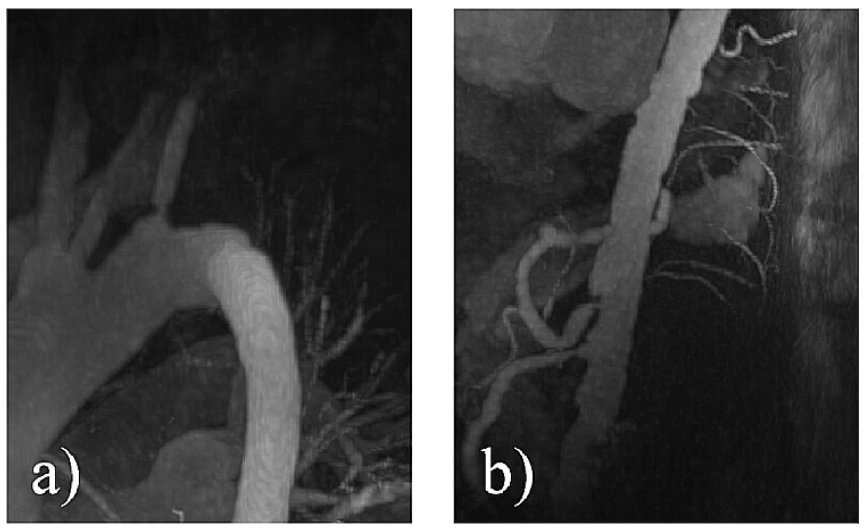

Fig. 11. Enlarged regions of Fig. 10 show stenosis of subclavian artery (a) and celiac trunk (b). The rough border of the aorta in (b) is consistent with significant arteriosclerosis.

The significance of aortic dissections was also studied by determining which organs were fed by the true or false lumen. Fully interactive 3-D manipulation was demonstrated without reduction in image resolution while the cine animation loop played at its full frame rate.

\section{DISCUSSION}

The multiple-echo trajectory allows acquiring four radial lines per TR instead of the two radial lines previously acquired [10] with only a $16 \%$ increase in repetition time (4.4 versus $3.8 \mathrm{~ms})$. The reduction in undersampling artifact allows a reduction in the breath-hold interval from $40 \mathrm{~s}$ to $30 \mathrm{~s}$ from the previous implementation. This reduction in the undersampling 
artifact reduces the structured background level and accounts for the wider variation in measured SNR improvement then one would simply expect from doubling the acquisition time. Of course, longer scans can still be performed if capturing temporal processes of a longer duration is necessary.

The data acquisition interval is designed so that fat accrues $180^{\circ}$ of phase between the first and fourth sampling of the $\mathrm{k}$-space origin (2.2 $\mathrm{ms}$ at $1.5 \mathrm{~T})$ [29]. While fat signal cancels only at the oversampled lower spatial frequencies, the intensity of the abdominal wall, intraperitoneal fat and retroperitoneal fat is significantly reduced. The obscuring fat signal, decreased SNR, and decreased CNR in our first implementation required the image volumes to be rendered over relatively small subvolumes. The improvements described here allow MIP rendering to detect the desired vascular signal over volumes similar to conventional techniques. This improvement requires a level of $B_{0}$ homogeneity that is sometimes difficult to maintain across the entire abdomen. Methods to create field maps from the multiple echoes and correct for inhomogeneity have been developed previously [15].

The temporal filter generation algorithm produces considerably clearer vessel time courses than our previous implementation. These filters improve SNR and remove disturbing low frequency background variations between frames that undermined confidence in making a diagnosis. The iterative method produces the narrowest temporal window for lower spatial frequencies based on sampling density. This in turn removes inconsistencies caused by parenchyma which enhancing only later in the scan. While the new algorithm removes many of the empirical elements in the design, further analysis is still necessary on designing the $F_{n}(t)$ function according to other physiological processes of interest outside the chest and abdomen.

The 3D PR trajectory incurs a SNR penalty of approximately $25 \%$ relative to Cartesian scanning for static objects [12] since its nonuniform sampling density is less efficient for decreasing noise relative to a uniform sampling density. Measuring SNR performance for time-variable intensities is more difficult to quantify because SNR will depend on the intensity variation during the scan. For Cartesian scans, the interval between peak intensity and where the center of k-space is imaged will also be crucial. As we have seen in the patient exams shown here, the peak enhancement time can vary dramatically. The temporal filters described here do attenuate data that would improve the SNR of static structures. However, the further loss of SNR is minimal because the filters suppress signal that would lower the variance of the relatively small volume of oversampled $k$-space, approximately $7 \%$ of the k-space sphere in this protocol.

Likewise, the peak time frame for a time-variable object often shows a SNR improvement compared to the time-averaged reconstruction, with the degree again depending on the shape and time-delay of enhancement. In the limit, the $F_{n}(t)$ can be designed as a matched filter for a specific enhancement pattern.

The relatively small volume of oversampled $\mathrm{k}$-space allows good visualization of flow patterns through larger vessels while less temporal information as vessels become smaller. However, even large vessels, such as the aorta in Fig. 7, show some artifactual temporal enhancement, however. Inspecting the source images demonstrates that the temporal filter, which widens with higher spatial frequencies, causes the edges of the aorta to enhance. Sagittal or coronal MIP projections of the enhanced vessel edges then appear as if the entire vessel lumen has enhanced. Efforts to increase the length of the echo train are ongoing and will allow the temporal filter to narrow and depict more temporal information. Normally longer data acquisition intervals increase concerns about off-resonant phase errors. However, off-resonance correction can be done after generating low-resolution field maps using the oversampled data. Also, methods for exploiting coil sensitivity in 2-D radial acquisitions [30] are being developed to suppress undersampling artifacts for 3D PR [31]. As the number of receiver coils grows on MRI scanners, the opportunity to synthesize unacquired data and narrow the temporal filter to provide additional temporal information will be possible. Finally, with wide variations possible for contrast arrival time, we have recently developed a 3-D fluoroscopic triggering method [32]. In real-time imaging, interesting opportunities exist to develop causal versions of the noncausal filters developed here.

\section{CONCLUSION}

The introduction of multiple-echoes has increased the sampling efficiency of VIPR and, thus, allowed higher SNR exams, shorter breath-holds, and higher CNR due to less undersampling artifact. An iterative density compensation algorithm provides simplified and improved temporal resolution with increased SNR relative to an empirically designed filter. With the addition of a distributed visualization system, users can interactively select a subvolume of the anatomy and view flow patterns through this region. This capability has proven very worthwhile when studying vasculature conditions such as dissections, aneurysms, complex and retrograde flow. Significant opportunities also exist to utilize this technique to simplify MRA while providing more comprehensive exams.

\section{ACKNOWLEDGMENT}

The authors would like to thank L. Flynn, K. Hellenbrand, and S. Fuller for their assistance in imaging the volunteers and patients.

\section{REFERENCES}

[1] J. P. Earls, N. M. Rofsky, D. R. DeCorato, G. A. Krinsky, and J. C. Weinreb, "Breath-hold single-dose gadolinium-enhanced three-dimensional MR aortography: usefulness of a timing examination and MR power injector," Radiology, vol. 201, no. 3, pp. 705-710, 1996.

[2] T. F. Hany, G. C. McKinnon, D. A. Leung, T. Pfammatter, and J. F. Debatin, "Optimization of contrast timing for breath-hold three-dimensional MR angiography,' J. Magn. Reson. Imag., vol. 7, no. 3, pp. 551-556, 1997.

[3] T. K. Foo, M. Saranathan, M. R. Prince, and T. L. Chenevert, "Automated detection of bolus arrival and initiation of data acquisition in fast, three-dimensional, gadolinium-enhanced MR angiography," Radiology, vol. 203, no. 1, pp. 275-280, 1997.

[4] A. H. Wilman, S. J. Riederer, B. F. King, J. P. Debbins, P. J. Rossman, and R. L. Ehman, "Fluoroscopically triggered contrastenhanced three-dimensional MR angiography with elliptical centric view order: application to the renal arteries," Radiology, vol. 205, no. 1, pp. 137-146, 1997.

[5] T. J. Carroll, F. R. Korosec, G. M. Petermann, T. M. Grist, and P. A. Turski, "Carotid bifurcation: evaluation of time-resolved three-dimensional angiography," Radiology, vol. 220, pp. 525-532, 2001. 
[6] T. F. Hany, T. J. Carroll, R. A. Omary, E. Esparza-Coss, F. R. Korosec, C. A. Mistretta, and T. M. Grist, "Contrast-enhanced, single-injection MRA with automated table movement compared to multi-injection, time-resolved CE 3D-MRA of the aorta and run-off vessels-initial results," Radiology, vol. 221, pp. 266-273, 2001

[7] J. P. Finn, V. Baskaran, J. C. Carr, R. M. McCarthy, F. S. Pereles, R. Kroeker, and G. A. Laub, "Thorax: low-dose contrast-enhanced threedimensional MR angiography with subsecond temporal resolution-initial results," Radiology, vol. 224, no. 3, pp. 896-904, 2002.

[8] M. Goyen, G. Laub, M. Ladd, J. Debatin, J. Barkhausen, K. Truemmler, S. Bosk, and S. Ruehm, "Dynamic 3D MR angiography of the pulmonary arteries in under four seconds," J. Magn. Reson. Imag., vol. 13, pp. 372-377, 2001.

[9] F. R. Korosec, R. Frayne, T. M. Grist, and C. A. Mistretta, "Time-resolved contrast-enhanced 3D MR angiography," Magn. Reson. Med., vol. 36, no. 3, pp. 345-351, 1996.

[10] A. V. Barger, W. F. Block, Y. Toropov, T. M. Grist, and C. A. Mistretta, "Time-resolved contrast-enhanced imaging with isotropic resolution and broad coverage using an undersampled 3D projection trajectory," Magn. Reson. Med., vol. 48, pp. 297-305, 2002.

[11] D. C. Peters, F. R. Korosec, T. M. Grist, W. F. Block, K. K. Vigen, J. E. Holden, and C. A. Mistretta, "Undersampled projection reconstruction applied to MR angiography," Magn. Reson. Med., vol. 43, pp. 91-101, 2000.

[12] C. M. Tsai and D. G. Nishimura, "Reduced aliasing artifacts using variable-density k-space sampling trajectories," Magn. Reson. Med., vol. 43, pp. 452-458, 2000

[13] P. Joseph and J. Whitley, "Experimental simulation of ECG-gated heart scans with a small number of views," Med Phys, vol. 10, pp. 444-449, 1983.

[14] C. M. Lai and P. C. Lauterbur, "True three-dimensional image reconstruction by nuclear magnetic resonance zeugmatography," Phys Med Biol, vol. 26, pp. 851-856, 1981.

[15] E. K. Brodsky, A. Lu, F. J. Thornton, T. M. Grist, and W. F. Block, "Using multiple half-echos to improve sampling efficiency and fat suppression in time-resolved MRA," in Proc. ISMRM 11th Annu. Meeting, Toronto, ON, Canada, 2003, p. 322.

[16] A. Lu, E. K. Brodsky, T. M. Grist, and W. F. Block, "Rapid fat suppressed isotropic steady-state free precession imaging using true 3D multiplehalf-echo projection reconstruction," Magn. Reson. Med., vol. 53, pp. 692-699, 2005.

[17] M. L. Lauzon and B. K. Rutt, "Effects of polar sampling in kspace," Magn. Reson. Med., vol. 36, no. 6, pp. 940-949, 1996.

[18] K. Scheffler and J. Hennig, "Reduced circular field-of-view imaging," Magn. Reson. Med., vol. 40, pp. 474-480, 1998.
[19] J. D. O'Sullivan, "A fast sinc gridding algorithm for Fourier inversion in computer tomography," IEEE Trans. Med. Imag., vol. MI-4, pp. 200-207, 1985.

[20] J. G. Pipe and P. Menon, "Sampling density compensation in MRI: rationale and an iterative numerical solution," Magn. Reson. Med., vol. 41, pp. 179-186, 1999.

[21] J. I. Jackson, C. H. Meyer, D. G. Nishimura, and A. Macovski, "Selection of a convolution function for Fourier inversion using gridding," IEEE Trans. Med. Imag., vol. 10, no. 3, pp. 473-478, Sep. 1991.

[22] J. G. Pipe, "Reconstructing MR images from undersampled data: dataweighting considerations," Magn. Reson. Med., vol. 43, pp. 867-875, 2000.

[23] — "Motion correction with PROPELLER MRI: application to head motion and free-breathing cardiac imaging," Magn. Reson. Med., vol. 42, pp. 963-969, 1999.

[24] H. K. Song and L. Dougherty, "K-space weight imaged contrast (KWIC) for contrast manipulation in projection reconstruction MRI," Magn. Reson. Med., vol. 44, pp. 825-832, 2000.

[25] M. I. Altbach, E. K. Outwater, T. P. Trouard, E. A. Krupinski, R. J. Theilmann, A. T. Stopeck, M. Kono, and A. F. Gmitro, "Radial fast spin-echo method for T2-weighted imaging and T2 mapping of the liver," J Magn. Reson. Imag., vol. 16, no. 2, pp. 179-189, 2002.

[26] V. Rasche, R. W. de Boer, D. Holz, and R. Proksa, "Continuous radial data acquisition for dynamic MRI," Magn. Reson. Med., vol. 34, pp. 754-761, 1995.

[27] M. J. Redmond, E. K. Brodsky, Y. H. Hu, T. M. Grist, M. J. Schulte, and W. F. Block, "The 4D cluster visualization project," in Proc. SPIE Int. Symp. Medical Imaging, San Diego, CA, 2004, pp. 28-38.

[28] E. K. Brodsky and W. F. Block, "Interactive visualization of time-resolved Contrast-Enhanced Magnetic Resonance Angiography (CE-MRA)," in IEEE Visualization, Seattle, 2003.

[29] A. C. Larson, O. P. Simonetti, and D. Li, "Coronary MRA with 3D undersampled projection reconstruction TrueFISP," Magn. Reson. Med., vol. 48, no. 4, pp. 594-601, 2002.

[30] M. A. Griswold, R. M. Heidemann, and P. M. Jacob, "Direct paralle imaging reconstruction of radially sampled data using GRAPPA with reduced shifts," in Proc. ISMRM 11th Annu. Meeting, Toronto, ON, Canada, 2003, p. 2349.

[31] A. Arunachalam, A. Lu, E. K. Brodsky, and W. F. Block, "GRAPPA for the 3D radial trajectory," in Proc. ISMRM 13th Annu. Meeting, Miami, FL, 2005, p. 2677.

[32] E. K. Brodsky, T. M. Grist, and W. F. Block, "Real-time 3D non-Cartesian phased-array contrast-enhanced MRA," in Proc. ISMRM 13th Annu. Meeting, Miami, FL, 2005, p. 379. 